\title{
Photon Count Nonlinearity Correction for Experimental Data Processing on Pulse Exited Optically Stimulated Luminescence of YAP:Mn
}

\author{
D. Afanassyev, S. Ubizskii, \\ R\&D Center for Solid State Electronics and Sensors \\ Lviv Polytechnic National University \\ Lviv, Ukraine \\ e-mail: denisna64@gmail.com
}

\author{
V. Rabyk, V. Paslavsky \\ Department of Electronics and Computer Science \\ Ivan Franko National University of Lviv \\ Lviv, Ukraine \\ e-mail: rabykv@ukr.net
}

\begin{abstract}
An approach to photon count nonlinearity correction for recording the response of optically stimulated luminescence excited by individual pulses is described. The pulse pair resolution parameter for dead time correction at high count rate is extracted from the set of recorded decay curves fitted with the Becquerel decay function. A substantial narrowing of the spread of normalized decay responses is achieved.
\end{abstract}

Index Terms - photon counting, pulse pair resolution, dead time correction, Becquerel decay function, optically stimulated luminescence (OSL), pulse mode OSL.

\section{INTRODUCTION}

The yttrium aluminum perovskite activated by $\mathrm{Mn}^{2+}$ (YAP: $\mathrm{Mn}^{2+}$ ) is considered a promising detector for passive OSL dosimetry [1,2] using the optically stimulated luminescence excited by short light pulses (P-OSL) for absorbed dose assessment [3]. The successful application of the P-OSL technique requires the development of dedicated instrumentation and OSL response readout method, taking into account peculiarities of the detector material and its OSL glow decay. The OSL of YAP: $\mathrm{Mn}^{2+}$ is not yet fully studied. The preliminary experiments have revealed its behavior considerably different from a simple exponential decay and lasts longer then it. To study OSL decay kinetics peculiarities it is desirable to measure the OSL response not only in a wide time scale but also in a wide dynamic range of photometric scale. The dynamic range of the light flux from the start of the decay process to the end of the time scale of interest (up to $500 \mathrm{~s}$ ) reaches several orders of magnitude. Therefore the accurate recording of such responses is complicated.

The light flux low end of the OSL response is so weak, that even the application of single photon counting technique here requires certain background reduction. The authors have successfully applied the synchronous background subtraction to tackle this problem obtaining the residual dark count below $20 \mathrm{~s}^{-1}$ for $1 \mathrm{~s}$ dwell time and even less for further increase in the latter $[4,5]$. From another side, the OSL emission intensity immediately after the excitation pulse in these experiments exceeded the linear range of photon counting, which is usually less than $10^{6} \mathrm{~s}^{-1}$. The measured photon count deviates from the linear dependence on input flux due to finite pulse pair resolution of the photomultiplier tube (PMT) together with the signal conditioning electronics (amplifier, comparator, counters), which introduces some dead time into data acquisition process. If the pulse pair resolution $\tau$ is known, the

This work was funded by Ministry of Education and Science of Ukraine under R\&D project «DB/Reader» (Ref. No. 0117U004443. count nonlinearity can be corrected by a simple expression $[6$, $7,8]$

$$
N=M /(1-M \cdot \tau),
$$

where $N$ and $M$ are the real and measured photon counting rate respectively. This expression is a linearized solution of the following equation $[6,8]$ :

$$
M=N \cdot \exp (-\tau \cdot N)
$$

For $\tau \cdot N<0.2$ the expressions (1) and (2) give sufficiently close results with residual error within $1 \ldots 2 \%$ [8], which may be comparable with noise spread. The linear response can therefore be extended up to about $10^{7} \mathrm{~s}^{-1}$ or even further [6], while using more elaborated model based on (2).

Such extension of dynamic range is enough for our experiment, but it needs a correct determination of $\tau$. The conventional procedure for measuring the pulse pair resolution involves the use of a calibrated light source or attenuator, which provides several different light intensities with known ratios [7] and is a major complication of the instrumentation. Fortunately it may be possible to extract the information on pulse pair resolution from the actually measured experimental data. This approach is based on assumption that the decay behavior of the OSL glow process under study remains the same after each excitation pulse over the whole range of photon counting rate from the highest one, which can be corrected, up to complete bleaching. This paper is devoted to testing of such approach for the measurement of the OSL response excited by the individual light pulse and checking the possibility to extend the dynamic range of OSL response measurement.

\section{EXPERIMENTAL}

The simplified arrangement of experiment is shown in Fig. 1. The apparatus is described in more detail in [5]. The OSL is excited by light pulses with a maximum wavelength at $\lambda=470$ $\mathrm{nm}$, generated by the high-intensity LED 1. Its excitation spectral range is further narrowed by an optional bandpass filter 2 installed before the sample under study 3 . The electromechanical shutter 4 allows the emission light to pass via another bandpass filter 5 to the detector 6 (Hamamatsu H9305-04 PMT module) only at $1 \mathrm{~s}$ long time intervals following the excitation pulses, after a fixed delay of $46 \mathrm{~ms}$. The electronics module contains pulse preamplifier, amplitude discriminator, counters, as well as the microcontroller, drivers 
and USB interface. The dedicated software runs on the $\mathrm{PC}$ to set all the parameters of the experiment, start the measurement procedure and record the data.

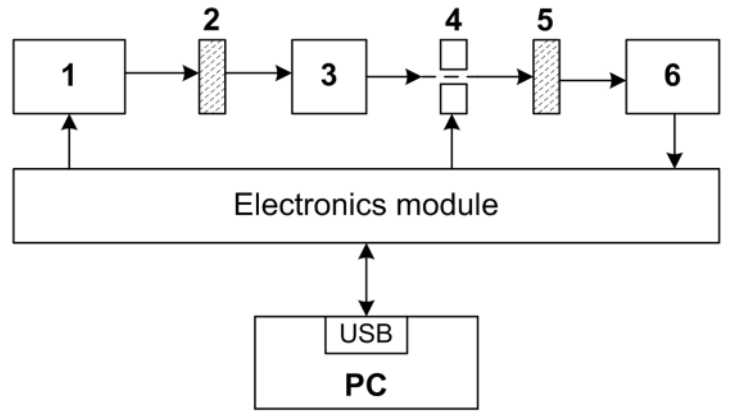

Figure 1. Experimental arrangement for recording the OSL response excited by individual light pulses.

The measurement procedure is modified in respect to one described in [5] to provide just one excitation pulse before the sequence of 128 time intervals of $1 \mathrm{~s}$ duration each, with repetition period of $3.1 \mathrm{~s}$ for response counting. Each $1 \mathrm{~s}$ interval is divided by 250 channels of $4 \mathrm{~ms}$ duration each, while identical $1 \mathrm{~s}$ intervals are provided for dark counts, accordingly to synchronous subtraction procedure described in [4]. The total duration of this recording was 397 s. It was considered enough for full elimination of OSL glowing after the single excitation pulse. The duration of each excitation pulse $100 \mathrm{~ms}$, and the LED current pulse amplitude was constant at $200 \mathrm{~mA}$. The measurement sequence for each excitation pulse was started manually. The time interval between subsequent pulses was kept constant at 7 minutes. Each OSL decay curve was recorded in a separate data file. The $1 \mathrm{~s}$ data intervals after application of the synchronous background subtraction procedure were merged together over the common time scale.

The samples were the $3 \times 3 \mathrm{~mm}^{2}$ polished square cuts of a YAP: $\mathrm{Mn}^{2+}$ single crystal annealed at $500{ }^{\circ} \mathrm{C}$ for $30 \mathrm{~min}$ and then irradiated by X-rays to the doses about 0.8 and 2 Gy. They were kept in dark all time after irradiation until the start of experiment. They were also preheated at $100{ }^{\circ} \mathrm{C}$ for 10 minutes before measurements.

\section{RESULTS AND DISCUSSION}

Several decay curves for OSL responses recorded on one of YAP:Mn samples are shown in Fig. 2.

It has been revealed that the most accurate fit for these data sets is a Becquerel decay function (BDF), known also as compressed hyperbola [9-11]:

$$
I(t)=a+b \cdot(1+c \cdot t)^{-1 / d}
$$

Where $a, b, c$ and $d$ are fitting parameters. The parameter $a$ corresponds to the constant bias, which in real condition can be due to the dark count of photometric system. It has no sense in the terms of decay process because it results in a divergence of photon counts at infinite time. Parameter $b$ describes the amplitude of decay curve. Parameter $c$ is responsible for the position of curve transition from nearly horizontal part to the sloped one in the double logarithm scale, and the parameter $d$ determines the general decay slope.

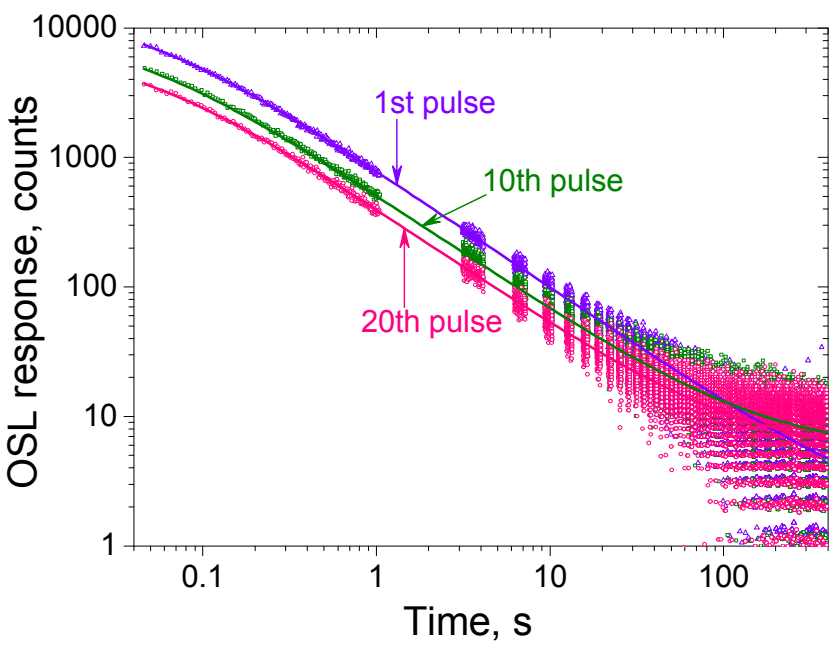

Figure 2. OSL decay curves (points) recorded after the 1st (violet), 10th (olive) and 20th (purple) sequential pulses together with their fits by mean of Becquerel decay function (1) (solid lines of corresponding color).

Fitted responses are also shown in Fig. 2. The sample was subjected to 20 subsequent excitation pulses resulting in a reduction of initial OSL response by factor of about 2 . Only responses for $1^{\text {st }}, 10^{\text {th }}$ and $20^{\text {th }}$ pulses are shown in Fig. 2 to demonstrate a trend.

One can expect that all parameters of BDF fit except an amplitude $b$ are to be the same for the unchanged shape of OSL kinetics, while the parameter $b$ will decrease exponentially with increase of number of excitation pulses. The real situation is somewhat different, although the decay curves shown in Fig. 2 look similar. The fit parameters $a, c$ and $d$ reveal monotonic changes from nonlinearity of photon counting at the initial part of measured signal when counting rate is close or exceeds $10^{-6} \mathrm{~s}^{-1}$, according to our assumption that the decay kinetics is the same for the whole sequence of excitation pulses. If this assumption is true, than the nonlinearity correction according to (1) with appropriate $\tau$ will bring all the decay curves to the same shape, i.e. fitting parameters $a, c$ and $d$ will coincide for all 20 pulses.

The pulse pair resolution parameter $\tau$ has been determined by brute force method. Its magnitude has been sought over a certain range of probable values. This range was initially set from 25 to $80 \mathrm{~ns}$ according to the prediction of pulse response behavior of both the PMT and the associated electronics. The $\tau=20 \mathrm{~ns}$ for PMT alone was estimated according to pulse response numbers form datasheet [7], while that for the counting circuit was previously determined in [4] being also under $20 \mathrm{~ns}$ by applying test pulse sequences from the oscillator. These two $\tau$ components added in series determine the estimation of 40ns for the cascaded deadtime for the complete signal processing system, according to [8]. Indeed, some uncertainty may be present in this estimation, so a range of $\tau$ values should be set including the above estimation and certain spread. The nonlinearity correction according to expression (1) with several values of $\tau$ was performed on 
measured OSL decay curves for the first two and the last two pulses in a set of experimental data. Then the BDF fit was applied to the corrected responses. The resulting $\tau$ has been determined by minimizing the mean square deviation between all mentioned four normalized fitting curves. The parameter $a$ has been omitted here for the reason described above. The finally adopted pulse pair resolution parameter was $\tau=35 \mathrm{~ns}$. This value is in good agreement with the expectations on the performance of this photon counting system. The result of this procedure is shown in Fig. 3 as a mean normalized decay curve of 20 sequential OSL responses. The error bar is $5 \%$ wide and shows the standard deviation of normalized fitted curves from the mean one over the whole set of 20 excitation pulses.

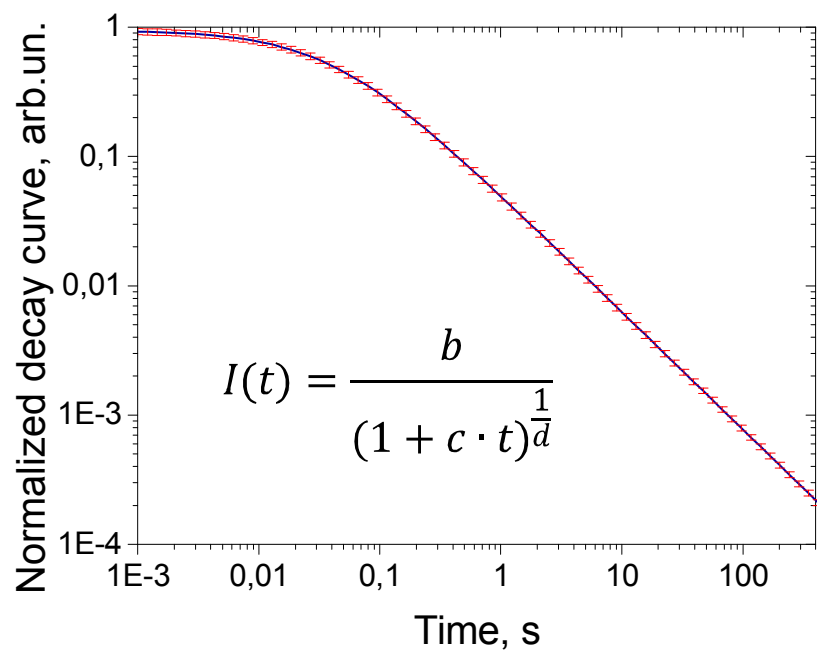

Figure 3. The averaged normalized BDF fits for all of 20 single pulse responses after nonlinearity correction with $\tau=35 \mathrm{~ns}$. Error bars height is about $5 \%$ of the signal value and corresponds to the standard deviation of the fit points of all 20 decay curves from the average one.

One should note that the behavior of parameter $a$ determined from BDF fit (3) after nonlinearity correction increases from near zero and saturates after several pulses at a level of about 15 counts for the sample shown in Fig. 2,3. It is manifested in Fig. 2 by somewhat elevated lower end of fitted decay curve from 10th and 20th pulses. We assume that this behavior can be explained by some residual even after $400 \mathrm{~s}$. This residual glow adds to the next excitation pulse producing some additional bias for the subsequent response.

\section{CONCLUSIONS}

The experiments on the pulse excited OSL carried out on single crystalline YAP:Mn dosimetric detectors have demonstrated that the decay measured over a wide dynamic range and time scale corresponds to the Becquerel decay function (compressed hyperbola). Assuming that the decay curve shape remains the same over the process of emptying the dosimetric traps we have derived the pulse pair resolution time parameter. This parameter made it possible to correct the nonlinearity of single photon count at high rate and to extend the dynamic range of light flux measurement. The further studies are necessary to understand the OSL decay kinetics peculiarities in the material under investigation and their physical meaning.

\section{ACKNOWLEDGMENT}

Authors are grateful to A. Suchocki, M. Berkowski and Ya. Zhydachevskii for providing of YAP:Mn single crystal samples for investigation and their preparation as well as to A. Luchechko for valuable assistance in carrying out the experiments.

\section{REFERENCES}

[1] Ya. Zhydachevskii, A. Suchocki, M. Berkowski, and Ya. Zakharko, "Optically stimulated luminescence of $\mathrm{YAlO}_{3}: \mathrm{Mn}^{2+}$ for radiation dosimetry," Radiat. Meas., vol. 42, pp. 625-627, 2007.

[2] Ya. Zhydachevskii, A. Luchechko, D. Maraba, N. Martynyuk, M. Glowacki, E. Bulur, S. Ubizskii, M. Berkowski, and A. Suchocki, Timeresolved OSL studies of $\mathrm{YAlO}_{3}: \mathrm{Mn}^{2+}$ crystals," Radiat. Meas., vol. 94, pp. 18-22, 2016.

[3] E.G. Yukihara and S.W.S. McKeever, Optically Stimulated Luminescence: Fundamentals and Applications, John Wiley \& Sons Ltd., 2011.

[4] D. Afanassyev, S. Ubizskii, Ya. Zhydachevskii, and V. Rabyk, "Photon counting with synchronous background subtraction for time resolved optically stimulated luminescence readout," to be published.

[5] S. Ubizskii, D. Afanassyev, Ya. Zhydachevskii, A. Luchechko, and V. Rabyk, "Set-up for pulsed OSL readout of YAP:Mn dosimetric detectors," to be published.

[6] D. P. Donovan, J. A. Whiteway, and A. 1. Carswell, "Correction for nonlinear photon-counting effects in LIDAR systems," Applied Optics. Vol. 32, No. 33, p.6742, 1993.

[7] Hamamatsu Photon counting heads H12386 series, Hamamatsu Photonics Datasheet (see https://www.hamamatsu.com/resources/pdf/ etd/H12386 TPMO1073E.pdf).

[8] R. Jenkins, "Quantitative X-Ray Spectrometry, Second Edition," CRC Press, 1995, ch. 4.6.

[9] M.N. Berberan-Santos, E.N. Bodunov and B. Valeur, "Mathematical functions for the analysis of luminescence decays with underlying distributions: 2. Becquerel (compressed hyperbola) and related decay functions," Chemical Physics, vol. 317, pp. 57-62, 2005.

[10] M.N. Berberan-Santos, E.N. Bodunov and B. Valeur, "Luminescence Decays with Underlying Distributions of Rate Constants: General Properties and Selected Cases of Rate Constants: General Properties and Selected Cases," Springer Series on Fluorescence, vol. 4, pp. 67-103, 2007.

[11] L. Whitehead, R. Whitehead, B. Valeur and M.N. Berberan-Santos, "A simple function for the description of near-exponential decays: The stretched or compressed hyperbola," American Journal of Physics, vol. 77, pp. 173-179, 2009. 